\title{
Enseñar y aprender, los retos de la educación virtual
}

\author{
Rossana Fernández Quintana \\ fernández.quintana@hotmail.com \\ Universidad Nacional de Pilar
}

\section{RESUMEN}

Construir entornos virtuales de aprendizaje (EVA) los cuales acorten distancias emocionales, cognitivas y geográficas es un desafío que muchos docentes enfrentan en la actualidad. (Morado, Ocampo, 2019). El presente trabajo da cuenta de una experiencia de Acompañamiento Tecno-Pedagógico (ATP) a un grupo conformado por 120 docentes de educación superior de una universidad pública entre febrero del 2020 y julio del 2021, en la ciudad de Pilar, departamento de Neembucú. El objetivo de la investigación consistió en analizar el desarrollo de competencias digitales en docentes a través de la implementación de los entornos virtuales de aprendizaje. A través del método etnográfico, utilizando el cuestionario en línea, el relevamiento de expresiones de los participantes en observación como métodos de recolección de datos, se analizaron las vivencias de los docentes en su proceso de apropiación de la tecnología y de cambio de sus prácticas a partir de los significados que ellos le asignaron al proceso de adquisición de competencias digitales aplicables en la educación virtual.

Palabras claves: educación virtual; competencias digitales; entornos virtuales de aprendizaje 


\title{
Teaching and learning, the challenges of virtual education
}

\begin{abstract}
Building virtual learning environments (VLE) which shorten emotional, cognitive and geographic distances is a challenge that many teachers face today. (Morado, Ocampo, 2019). This work reports an experience of Techno-Pedagogical Accompaniment (ATP) to a group made up of 120 higher education teachers from a public university between February 2020 and July 2021, in the city of Pilar, department of Neembucú. The objective of the research was to analyze the development of digital skills in teachers through the implementation of virtual learning environments. Through the ethnographic method, using the online questionnaire, the survey of expressions of the participants in observation as data collection methods, the experiences of teachers in their process of appropriation of technology and change of their practices were analyzed. starting from the meanings they assigned to the process of acquiring digital skills applicable in virtual education.
\end{abstract}

Keywords: virtual education; digital skills; virtual learning environments.

Artículo recibido: 10 Setiembre. 2021 Aceptado para publicación: 15 Octubre. 2021 Correspondencia: fernández.quintana@ hotmail.com Conflictos de Interés: Ninguna que declarar 


\section{INTRODUCCIÓN}

La educación a distancia en tiempos de pandemia del Covid-19 paso de ser una alternativa a una imperiosa necesidad para dar continuación a los planes de clases propuestos a inicios del año 2020, para ello fue necesario recurrir a las ya conocidas herramientas digitales y su aplicación en el ámbito educativo, pero ya no como apoyo a las clases presenciales, sino como, temporalmente, única forma de enseña y aprender.

La contingencia ha puesto en evidencia que los docentes son uno de los factores más importantes en el proceso de enseñanza aprendizaje, sin embargo, no se le ha capacitado ya que la introducción del e-Learning en la educación va más allá de la introducción de las TICs en los procesos educativos. La aplicación de las herramientas digitales en la educación virtual ha fomentado el aprendizaje online, rompiendo muchas barreras limitantes de la educación tradicional como el espacio, tiempo, cantidad y cobertura (Maraza, 2016).

El e-learning se ha consolidado como una opción de formación viable y de calidad frente a otras modalidades más tradicionales. Con el término e-learning se denomina, según (Domínguez y Marcelo, 2013) a aquella modalidad formativa que se desarrolla haciendo uso de los recursos electrónicos a través de redes y tecnologías de información y comunicación, traslada las experiencias educativas fuera de la tradicional aula de clases, esto es; aprendizaje en cualquier momento y en cualquier lugar, sin barreras geográficas o de agenda, utilizando el internet para el acceso a los materiales de aprendizaje e interactuando con expertos y estudiantes semejantes.

En virtud a estos importantes cambios como consecuencia del avance tecnológico y la necesidad de realizar actividades de forma remota, los docentes se ven en la obligación de actualizar sus perfiles a fin de lograr adquirir habilidades y destrezas en el ámbito tecnológico, y así lograr llevar a cabo de manera fluida y efectiva, la aplicabilidad de las herramientas digitales, todo ello acorde a los nuevos lineamientos del sistema educativo, pues ya no se reduce solo a que los docentes conozcan y manejen equipos tecnológicos sino que también le den el uso adecuado como complemento de sus clases habituales.

El objetivo de este trabajo se centra en el análisis del desarrollo de competencias digitales en docentes del Colegio San Lorenzo específicamente los cursos comprendidos desde el primero hasta el tercero de la educación media, a través de la implementación de los entornos virtuales de aprendizaje, para ellos es preciso conocer sobre la capacitación de 
los docentes en aplicación de herramientas digitales en la educación y relacionarla a la implementación de las herramientas digitales en el desarrollo de las clases virtuales síncronas y asíncronas.

\section{REVISIÓN BIBLIOGRÁFICA}

\subsection{La educación en línea}

La educación a distancia ha pasado por diferentes etapas a través de los años, su evolución ha sido vertiginosa ya que al introducir las tecnologías estas han ido ampliando el concepto. La aparición masiva de los medios digitales y las tecnologías que se utilizan en la educación produjo un despliegue plural de términos de términos tales como: educación distribuida, aprendizaje electrónico (e-learning), educación virtual, educación en línea, aprendizaje combinado (Blended-Learning), aprendizaje móvil (m-learning) (Verdún, 2016). Todo este auge de tecnologías enriquece el sistema de educación a distancia, por lo que consideramos importante describir algunas de estas modalidades.

La educación en línea en inglés e-learning, primeramente, se concebía como aquella que involucra cualquier medio electrónico de comunicación, incluyendo la videoconferencia y la audioconferencia. En sentido más específico, la educación en línea significa enseñar y aprender a través de computadoras conectadas en red. No se define en oposición a lo presencial, son espacios creados con y a partir de la tecnología, "la tecnología es el espacio mismo, es el territorio en el que se desenvuelven las acciones educativas". (Schwartzman, Tarasow, Trech, 2019). No obstante, algunos autores las traducen literalmente como un aprendizaje electrónico que refiere a algún tipo de proceso de enseñanza-aprendizaje realizado con ordenadores conectados a Internet y otras nuevas tecnologías móviles de telecomunicaciones. A través del tiempo se han ido incorporando nuevas tecnologías para la enseñanza y el aprendizaje, llegando a pensar que todo cambiaria, sin embargo las posibilidades del e-learning no se agotan con la incorporación de una tecnología o accesorio más al proceso de enseñanza, sino que adopta un modo muy diferente de concebir la comunicación, la interacción y las acciones tendientes a promover la comprensión entre los sujetos (Verdún, 2016).

Para el m-Learning o aprendizaje móvil, no hay una definición unánimemente aceptada, aunque todas ellas hacen referencia al uso de dispositivos móviles para facilitar el aprendizaje en cualquier momento y en cualquier lugar (López, Silva, 2016). Para García (2018) es un concepto que se utiliza en el ámbito de la educación a distancia para indicar 
que, mediante tecnología digital de ciertos equipos, es posible obtener experiencias educativas desde cualquier parte y en el momento preciso en que lo desee un estudiante. La tecnología mencionada se hace posible gracias a la transmisión de datos por el sistema WiFi. Para Verdún (2016) el aprendizaje móvil es una posibilidad de aprender a través de Internet, de diversas plataformas tecnológicas, pero con la máxima portabilidad, interactividad y conectividad. De cualquier forma, no podemos discutir que los dispositivos móviles son una herramienta muy utilizada por los estudiantes en su proceso de aprendizaje, manteniéndolos es constante comunicación con los involucrados en su educación. El teléfono celular es el dispositivo más utilizado y el preferido de los jóvenes en este tipo de aprendizaje; desde el cual pueden acceder a diferentes recursos y cada día existen en el mercado celulares con mayor capacidad y funciones que antes no se tenían. La educación en línea posee una especie de fuerza centrípeta que hace converger a todos los actores del proceso educativo en un espacio central común, donde ya no hay distancia (Schwartzman, Tarasow, Trech, 2019).

\subsubsection{Beneficios de la educación en línea}

En la formación por medios virtuales se permite el trabajo de las competencias correspondientes a cada asignatura, pero también se desarrollan competencias generales en el alumno como la planificación y gestión del tiempo, la comunicación oral y escrita en la propia lengua, habilidades informáticas básicas, el desarrollo de habilidades, de investigación, etc. (Alonso, Blázquez, 2016). Los beneficios de la educación en línea, según (Cadena, Sarmient, Casanova, 2020) son:

- Extender y facilitar el acceso a la formación a colectivo e individuos que no puedan acceder a la modalidad presencial.

- Incrementar la autonomía y responsabilidad del estudiante en su propio proceso de aprendizaje.

- Superar las limitaciones provocadas por la separación en espacio y/o tiempo del profesor-alumnos. - Gran potencial interactivo entre profesor-alumno.

- Flexibilidad en los tiempos y espacios educativos.

- Acceder a multiplicidad de fuentes y datos diferentes de los ofrecidos por el profesor en cualquier momento y desde cualquier lugar.

- Aprendizaje colaborativo entre comunidades virtuales de docentes y estudiantes.

- Optimización de los recursos 
- Ahorro de tiempo

- Aprendizaje autodirigido

- Disponibilidad permanente de archivo acumulativo de contenido del curso y actualizaciones oportunas.

- Estandarización de programas, etc.

\subsection{Los estudiantes en línea}

Los estudiantes del Siglo XXI han experimentado un cambio radical con respecto a sus inmediatos predecesores. No se trata sólo de las habituales diferencias en argot, estética, indumentaria y ornamentación personal o, incluso, estilo, que siempre quedan patentes cuando se establece una analogía entre jóvenes de cualquier generación respecto a sus antecesores, sino que nos referimos a algo mucho más complejo, profundo y trascendental: se ha producido una discontinuidad importante que constituye toda una "singularidad"; una discontinuidad motivada, sin duda, por la veloz e ininterrumpida difusión de la tecnología digital, que aparece en las últimas décadas del Siglo XX. Los universitarios de hoy constituyen la primera generación formada en los nuevos avances tecnológicos, a los que se han acostumbrado por inmersión al encontrarse, desde siempre, rodeados de ordenadores, vídeos y videojuegos, música digital, telefonía móvil y otros entretenimientos y herramientas afines. En detrimento de la lectura (en la que han invertido menos de $5.000 \mathrm{~h}$ ), han dedicado, en cambio, $10.000 \mathrm{~h}$ a los videojuegos y $20.000 \mathrm{~h}$ a la televisión, por lo cual no es exagerado considerar que la mensajería inmediata, el teléfono móvil, Internet, el correo electrónico, los juegos de ordenador... son inseparables de sus vidas. Resulta evidente que nuestros estudiantes piensan y procesan la información de modo significativamente distinto a sus predecesores. Además, no es un hábito coyuntural sino que está llamado a prolongarse en el tiempo, que no se interrumpe sino que se acrecienta, de modo que su destreza en el manejo y utilización de la tecnología es superior a la de sus profesores y educadores.

\subsubsection{Capacidad de atención de los estudiantes.}

Los nativos digitales se identifican con la interactividad: una respuesta inmediata a todas y cada una de sus acciones. No hay duda de que la escuela tradicional ha ofrecido muy poco en este sentido, en comparación con el resto de su mundo. 
Un estudio llegó a demostrar que los alumnos hacen una pregunta en clase cada diez horas. Por lo tanto, de manera general, no es que los Nativos de la era digital no sean capaces de prestar atención, sino que eligen no hacerlo.

Una investigación realizada por "Barrio Sésamo" pone de manifiesto que los niños, en realidad, no ven la televisión continuamente, sino "a ráfagas": sintonizan lo justo para captar lo esencial y asegurarse de que tiene un sentido. En otro experimento clave, a la mitad de un grupo de niños de cinco años se les puso un programa de televisión en un cuarto lleno de juguetes, y a la otra mitad se les puso el mismo programa televisivo, pero en una habitación sin ningún entretenimiento. Como era de esperar, el grupo que tenía cerca los juegos se distraía y veía el programa sólo el $47 \%$ de su tiempo, frente al $87 \%$ del grupo que no tenía a mano los juguetes. Sin embargo, cuando se les hizo preguntas acerca de lo que recordaban y entendían, los resultados fueron exactamente los mismos. Llegamos a la conclusión de que los niños de cinco años que formaron parte del grupo que tenía cerca los juguetes atendió estratégicamente, distribuyendo su atención e interés entre el juguete y el programa, de manera que sólo atendían a lo que era más sugerente para ellos. La estrategia instintiva que emplearon fue tan eficaz que los niños no pudieron tener más información, habiendo atendido más.

\subsection{Plataforma Moodle}

Es una de las plataformas más populares, surge como el resultado del trabajo de varios desarrolladores que trabajaron en un código abierto que lo han convertido en una plataforma de fácil uso. Moodle es un sistema para el Manejo del Aprendizaje en línea gratuito, que les permite a los educadores la creación de sus propios sitios web privados, llenos de cursos dinámicos que extienden el aprendizaje, en cualquier momento, en cualquier sitio (Moodle.org, 2017) destaca que puede cumplir las necesidades tanto para docentes como para estudiantes o si se es un administrador de la plataforma. Entre las características que ofrece se citan las siguientes:

- Posee una interfaz fácil de usar de tal forma que no existan dificultades para manejarla sea en computadoras de escritorio o dispositivos móviles.

- Posee un tablero personalizado que muestra de forma organizada los cursos disponibles así como la organización de los temas que diseñe el maestro.

- Presenta herramientas y actividades colaborativas como foros, wikis, talleres, etc. 
- Posee un calendario para el control de entrega de trabajos en fechas y tiempos establecidos.

- Gestión eficiente de los archivos.

- Editores de textos simples con formatos agradables para el usuario.

- Manejo de notificaciones sobre el acceso a la plataforma. Incluso identificación de tiempos de conexión de usuarios.

- Monitoreo del progreso de los cursos.

\subsection{Metodología de Aula invertida}

En casa los estudiantes estudian y se preparan para participar en las actividades. En clase son los estudiantes quienes practican aplicando conceptos claves mientras reciben retroalimentación del profesor o algún compañero. Después de la clase, son los mismos estudiantes quienes evalúan su entendimiento y a la vez extienden su aprendizaje.

Partiendo de las aseveraciones anteriores, en el aula invertida se transforma tanto la labor del estudiante como del docente, generando la necesidad de involucrar a los primeros para ser gestores de su propio aprendizaje autónomo fuera del aula, y se busca capacitar a los segundos para dirigir a sus estudiantes a través de procesos formativos con componentes virtuales asincrónicos.

La implementación de un modelo de aula invertida supone la creación o selección de diversos materiales de estudio para entregar a los estudiantes, de forma que estos realicen la exploración previa requerida.

Flores (2015), advierte que estos contenidos deben cumplir con los objetivos de aprendizaje del proceso formativo, su selección depende exclusivamente del docente, es fundamental que éste desarrolle sentido crítico que le permita definir qué contenido puede y debe abordarse en el aula, y qué material debe ser puesto a disposición de los estudiantes para el aprendizaje autónomo.

\section{METODOLOGÍA}

La metodología de investigación se basó en el método etnográfico, específicamente la etnografía virtual. La etnografía virtual tiene su origen cuando los investigadores comienzan a mostrar interés ante el fenómeno que representa la generación de espacios de socialidad, producto de la configuración de comunidades virtuales en Internet; una tecnología a través de la cual se favorece la emergencia de canales de comunicación que potencian la interactividad, tanto asincrónica como sincrónica a través de correos 
electrónicos, las wikis, las redes sociales, los foros, las redes de colaboración, e incluso plataformas educativas donde se usan otros recursos como aulas virtuales, videoconferencias o la mensajería instantánea (Ruiz, Aguirre, 2012).

Se analizaron las expresiones de los docentes, recolectadas a través de la técnica de cuestionario en línea y el análisis de lo expresado en los foros en el entorno virtual de sus clases desarrolladas, los cuales permitieron conocer los significados que el grupo de docentes dio a sus prácticas en clase.

Los instrumentos de recolección de datos empleados fueron: cuestionarios en línea enviados a los docentes y bitácora de observación durante los encuentros presenciales con los docentes. El proceso de investigación y recolección de datos fue comunicado a los docentes desde la presentación del trabajo.

\section{RESULTADOS Y DISCUSIÓN}

\subsection{Capacitación de docentes en aplicación de herramientas digitales en la educación.}

Los resultados representados en la Figura $\mathrm{N}^{\circ} 1$ indican que los docentes presentan mejores puntuaciones en el conocimiento de programas de edición de textos, puesto que estos programas son utilizados por los mismos cotidianamente en la aplicación de las clases presenciales, las aulas virtuales son conocidas y utilizadas por los docentes como herramienta principal para el desarrollo de las clases virtuales, así también conocen las formas de almacenamiento de archivos en internet porque es una herramienta incorporada a las aulas virtuales utilizadas comúnmente. 
Figura $\mathbf{N}^{\circ}$ 1: Plataformas aplicables a la educación virtual.

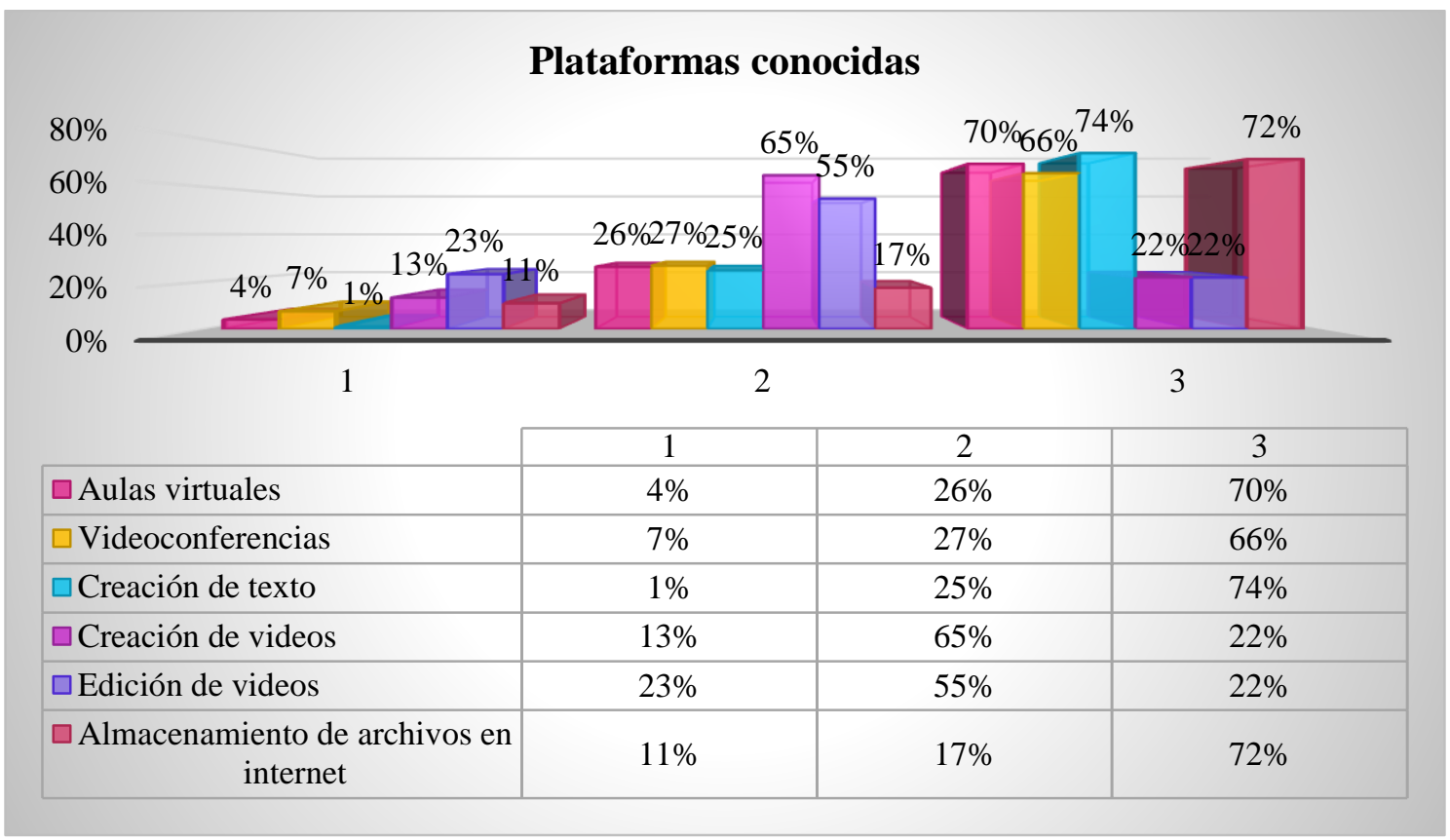

Fuente: Elaboración propia a partir de datos obtenidos del cuestionario en línea y observación etnográfica

Se presentaron puntajes menos favorecedores en el contenido multimedia, con dificultades por parte de los docentes para la elaboración de videos desde la grabación de los mismas y la posterior edición, es ese contexto las videoconferencias resultan más eficientes debido a la sincronicidad de la grabación de los videos en colaboración de los estudiantes.

La utilización del e-learning en los procesos de enseñanza y aprendizaje, con la incorporación de las herramientas tecnológicas, permite la formación de los educandos sin la restricción del espacio y el tiempo (Rodenes, Vallés, Moncaleano, 2013). Los docentes requieren de conocimientos básicos de varias herramientas para poder imprentarlas en la educación virtual puesto que las Tecnologías de la Comunicación y la Información se constituyen como herramientas interactivas para educar, de manera tal que los actores puedan interactuar, comunicarse directamente, en un entorno o contexto colaborativo. Asimismo, los estudiantes van a poder autorregularse en su proceso de aprendizaje.

En cuanto a la efectividad de la enseñanza desde la modalidad de e-learning, más allá de la cantidad de tiempo que se permanezca conectado o en línea, lo importante es la utilidad que se encuentra en la cantidad de tiempo dedicado al aprendizaje interactivo (Castaño, 
Duart, Sancho, 2015).

En todo caso, en el e-learning la presentación de las actividades tiene un carácter flexible para el uso de las TIC. Dado que los materiales usados en los procesos de enseñanza y de aprendizaje pueden ser grabados y usados en cualquier momento. Asimismo, lo que corresponde a los blogs, las construcciones wiki y otros recursos afines estarán habilitados para que los estudiantes puedan hacer uso de ellos.

\subsection{Implementación de herramientas digitales en el desarrollo de las clases virtuales síncronas y asíncronas.}

La figura $\mathrm{N}^{\circ} 2$ indica puntuaciones bajas en términos de calidad de las clases virtuales síncronas, específicamente en la calidad de equipos informáticos y el entorno donde se desarrollan las clases. La totalidad de los docentes cuentan con computados pero no todas están equipadas con las cámaras, micrófonos y auriculares de calidad que permitan el desarrollo de las clases por medio de videoconferencias. El entorno es por lo general la residencia de los docentes, por lo que en ciertas clases carecen de luminosidad suficiente y el sonido de la voz del docente es solapado con el del entorno.

Se requiere de lugares destinados al desarrollo de las clases, y equipamientos profesionales que realcen la calidad de las mismas.

Figura $\mathbf{N}^{\circ}$ 2: Calidad de equipos informáticos y entorno de clases.

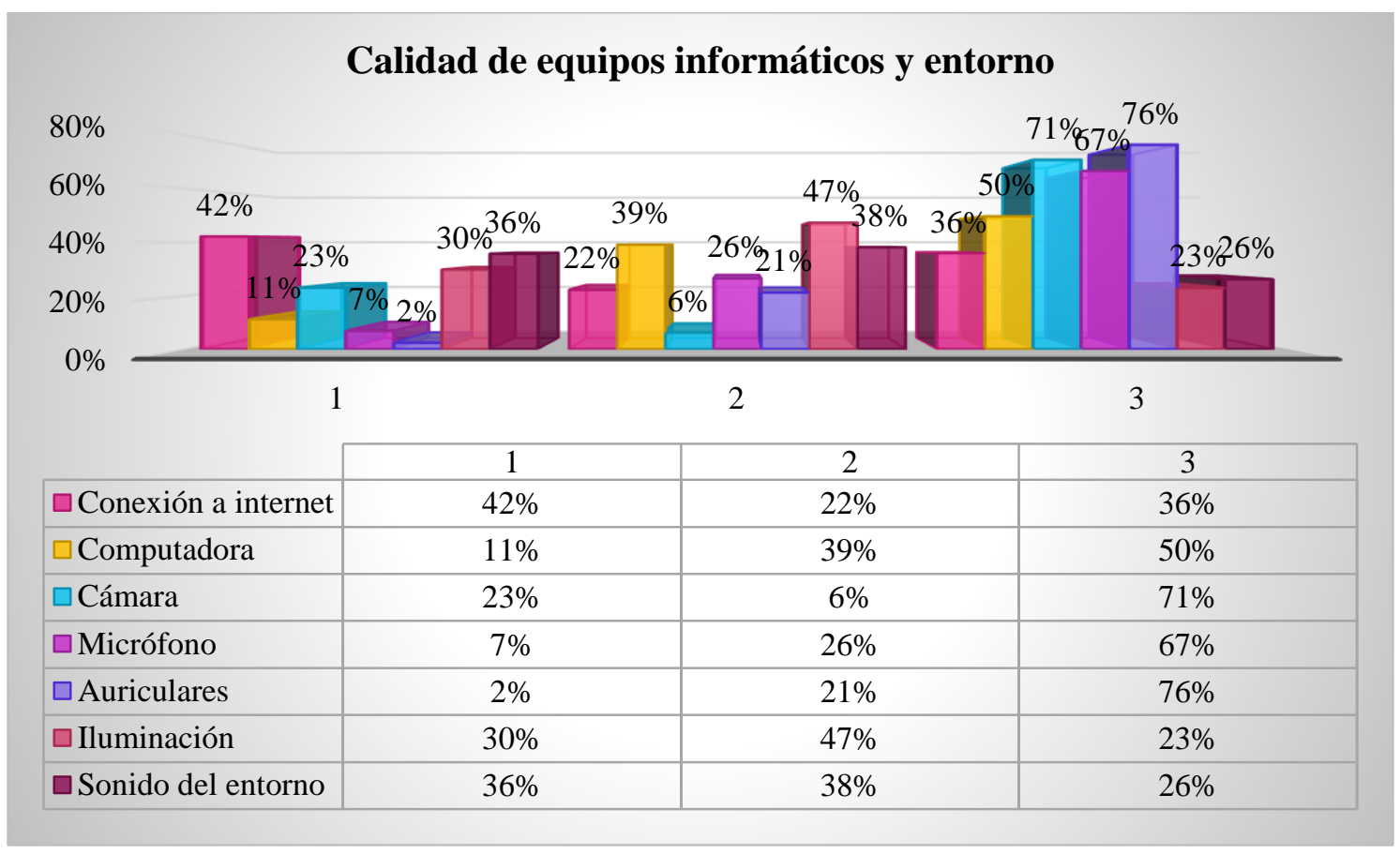

Fuente: Elaboración propia a partir de datos obtenidos del cuestionario en línea y observación etnográfica. 
Figura $\mathbf{N}^{\circ}$ 3: Materiales didácticos elaborados por los docentes

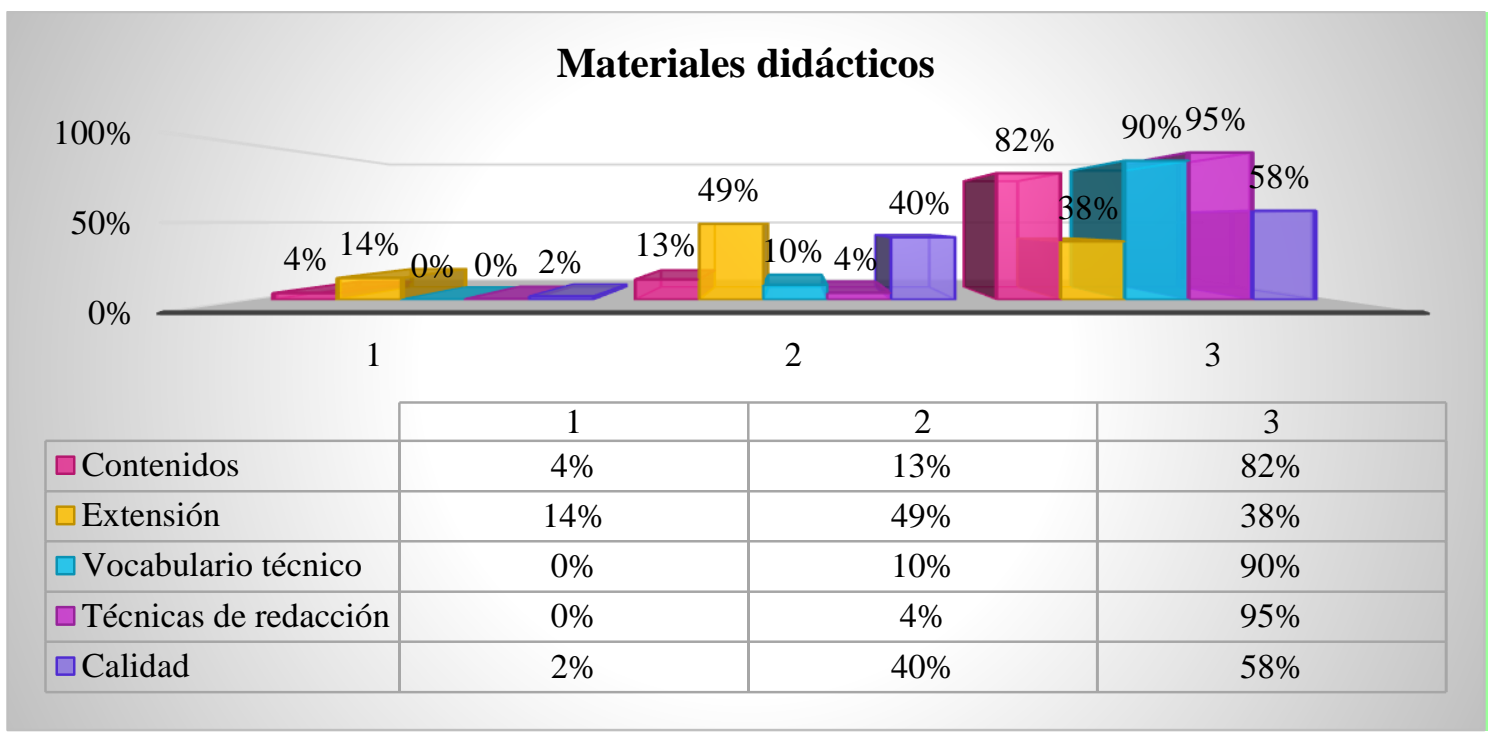

Fuente: Elaboración propia a partir de datos obtenidos del cuestionario en línea y observación etnográfica.

Los materiales didácticos preparados por los docentes, según sus mismos estudiantes y directivos son de buena calidad en cuanto al contenido, exceptuando la calidad de las imágenes contenidas por el número de pixeles y la edición de las mismas. El contenido referente a las materias es apropiado, en algunos casos se puede consideran un tanto extenso y en otros escueto.

\section{CONSIDERACIONES FINALES}

Los ambientes virtuales de aprendizaje tienen cada vez mayor preponderancia en el proceso de enseñanza. La incursión tecnológica se ha convertido en un reto para los modelos pedagógicos. Es por ello que surge la imperiosa necesidad de aplicar estrategias innovadoras que propicien el desarrollo de competencias que puedan fomentar en los estudiantes su capacidad crítica y reflexiva de conocimientos básicos en distintos ámbitos. Las practicas docentes anteriores a la implementación masiva de entornos virtuales debido al confinamiento por la pandemia del Covid-19 se basaban en metodología aplicables a clases presenciales donde la presencialidad era indispensable. Metodología adaptable para las clases síncronas, no así para las asíncronas donde se requiere de un mayor trabajo de elaboración de materiales de apoyo e instrumentos de evaluación.

Las prácticas educativas se construyen con el desarrollo de las competencias que ponen en práctica los docentes para la construcción y aplicación del conocimiento, acción que se realiza a partir de variables singulares. En este sentido es importante evaluar el tipo de 
competencias docentes que están desarrollando los profesores de educación media y los efectos de su enseñanza en el aprendizaje de sus alumnos, para reflexionar sobre acciones de capacitación que es necesario efectuar en la comunidad docente y mejorar así la práctica que incida de forma positiva en los estudiantes

Los docentes siguen en proceso de capacitación, proceso que no debe cortarse puesto que se hace necesaria dicha capacitación preferentemente no presencial, a través de plataformas tecnológicas, para que se posibilite y flexibilice el acceso y el tiempo en el proceso de enseñanza aprendizaje, así como lo descripto por (Zapata, 2013) capacitación que adecua las habilidades, necesidades y disponibilidades de cada discente, además de garantizar ambientes de aprendizaje colaborativos mediante el uso.

La aplicación de las herramientas digitales en la educación, para el caso de estudio, es parcial y gradual. Requiere apoyo académico y económico, puesto que se puede avanzar en implementación de clases síncronas y asíncronas, técnicas para los trabajos remotos, pero este avance debe ir acompañado de una buena cobertura de internet para los usuarios docentes y estudiantes y equipos informáticos.

(Ibarrola, 2020) afirma que es el momento para revisar las rígidas formas tradicionales de los contenidos nacionales, en particular su inflexible secuenciación. Al mismo tiempo que se exige la recuperación de las experiencias de todo tipo, de los aprendizajes logrados, que seguramente serán diferentes e incluso superiores a los transmitidos, es decir, se deben buscar nuevas formas y referentes para la evaluación diagnóstica de lo aprendido y su certificación. Se debe aprovechar la autonomía profesional de cada docente.

\section{REFERENCIAS BIBLIOGRÁFICAS}

Cadena, M., Sarmient, M., Casanova, J. (2020). Análisis de acceso a la educación ante la pandemia de COVID-19, en la educación Media Superior de la Universidad Autónoma de Campeche. Corporación Centro Internacional de Marketing Territorial para la Educación y el Desarrollo. (978-958-52748). Universidad Autónoma de Campeche. México.

Castaño, J., Duart, J. y Sancho, T. (2015). Determinantes del uso de internet para el aprendizaje interactivo: un estudio exploratorio. New approches in educational research, 4(1), 25-34.

Duart, J., y Martínez , M. (2001): "Evaluación de la Calidad docente en entornos virtuales de aprendizaje". 
García, R., Aguaded, I., Bartolomé, A. (2018). La revolución del blended learning en la educación a distancia.

Hamidian, B. (2010). Usos y necesidades de formación en tecnología de información y comunicación de los docentes de la Facultad de Ciencias Económicas y Sociales (Universidad de Carabobo). (Tesis doctoral inédita) Universidad de Sevilla.

León, L. (2017). Perfil de competencias del tutor virtual de la asignatura creatividad e inventiva. (Tesis de maestría). Universidad de Carabobo, Bárbula, Venezuela. Recuperado

de http://mriuc.bc.uc.edu.ve/bitstream/handle/123456789/4406/lleon.pdf?sequence $=1$

Maraza, B. (2016). Hacia un Aprendizaje Personalizado en Ambientes Virtuales. Campus virtuales, $\quad 5(1), \quad 20-29 . \quad$ Recuperado de https://dialnet.unirioja.es/servlet/articulo?codigo $=5400592$

Morado, M., Ocampo, S., (2019). Una experiencia de acompañamiento tecno-pedagógico para la construcción de Entornos Virtuales de Aprendizaje en educación superior. Educación [online]. 2019, vol.43, n.1， pp.43-61. ISSN 22152644. http://dx.doi.org/10.15517/revedu.v43i1.28457.

Prensky, M. (2010). Nativos e inmigrantes digitales: Adaptación al castellano del texto original "Digital Natives, Digital Immigrants". Cuadernos SEK 2.0-Madrid. España: Institución Educativa SEK Distribuidora SEK, S.A.

Ruiz, M., Aguirre, G., (2012). Etnografía virtual, un acercamiento al método y a sus aplicaciones. Estudios sobre las Culturas Contemporáneas, vol. XXI, núm. 41, pp. 67-96, 2015. Universidad de Colima.

Sáez, J., Domínguez, C., Mendoza, V. (2014). Valoración de los obstáculos, ventajas y prácticas del e-learning: un estudio de caso en Universidades Iberoamericanas. Education Siglo XXI, 32(2 Julio), 195-220. doi:http://dx.doi.org/10.6018/j/202221

Schwartzman, G., Tarasow, F. y Trech, M. (2019). De la educación a distancia a la educación en línea: aportes a un campo en construcción. Homo Sapiens Ediciones. https://elibro.net/es/ereader/uacam/112908?page $=18$

Verdún, N. (2016). Educación virtual y sus configuraciones emergentes: Notas acerca del elearning, b-learning y m-learning. Háblame de TIC, 3, 67-88. Recuperado de: 
https://www.uv.mx/blogs/brechadigital/files/2015/05/HdT3_Verd\%C3\%83\%C2 $\%$ BA n.pdf.

Zapata, M. (2013). MOOC, una visión crítica y una alternativa complementaria: La individualización del aprendizaje y de la ayuda pedagógica. Campus Virtuales. Revista científica iberoamericana de tecnología educativa. 1 (2), 20-38. 